\title{
ON THE UNIFICATION OF DWARF AND GIANT ELLIPTICAL GALAXIES
}

\author{
Alister W. Graham ${ }^{1}$ and Rafael Guzman ${ }^{1}$ \\ ${ }^{1}$ Department of Astronomy, University of Florida, Gainesville, FL 32611, USA.
}

\begin{abstract}
The near orthogonal distributions of dwarf elliptical (dE) and giant elliptical (E) galaxies in the $\mu_{e}-M$ and $\mu_{e}-\log R_{e}$ diagrams have been interpreted as evidence for two distinct galaxy formation processes. However, continuous, linear relationships across the alleged $\mathrm{dE} / \mathrm{E}$ boundary at $M_{B}=-18 \mathrm{mag}$ - such as the relationships between central surface brightness $\left(\mu_{0}\right)$ and: a) galaxy magnitude $(M)$; and b) light-profile shape $(n)$ - suggest a similar initial formation mechanism. Here we explain how these latter two trends in fact necessitate a different behavior for $\mathrm{dE}$ and $\mathrm{E}$ galaxies, exactly as observed, in diagrams involving $\mu_{e}$ (and also $\langle\mu\rangle_{e}$ ). A natural consequence is that the location of dEs and Es in Fundamental Plane type analyses that use $I_{e}$, or $\langle I\rangle_{e}$, will also be different. Together with other linear trends across the alleged $\mathrm{dE} / \mathrm{E}$ boundary, such as those between luminosity and color, metallicity, and velocity dispersion, it appears that the $\mathrm{dEs}$ form a continuous extension to the E galaxies. The presence of partially depleted cores in luminous $\left(M_{B}<-20.5 \mathrm{mag}\right)$ Es does however signify the action of a different physical process at the centers $(<\sim 300 \mathrm{pc})$ of these galaxies.
\end{abstract}

The common distinction between a dwarf elliptical (dE) galaxy and an (ordinary) elliptical (E) galaxy is whether the absolute magnitude is fainter or brighter than $M_{B}=-18 \mathrm{mag}$ respectively $\left(H_{0}=50 \mathrm{~km} \mathrm{~s}^{-1} \mathrm{Mpc}^{-1}\right.$, Sandage \& Binggeli 1984). By the term $\mathrm{dE}$, we additionally mean objects brighter than $-13 B$-mag; that is, we are not talking about (Local Group) dwarf spheroidal galaxies, whose range of colors suggest a range of formation processes (e.g., Conselice 2002). The realization that $\mathrm{dE}$ light-profiles could be reasonably well described with an exponential function (Faber \& Lin 1983; Binggeli, Sandage \& Tarenghi 1984) and that bright ellipticals are better fit with de Vaucouleurs' $r^{1 / 4}$-law helped lead to the notion that they are two distinct families of galaxies (e.g., Wirth \& Gallagher 1984, but see Graham 2002). One of the seminal papers supporting this view is Kormendy (1985). By plotting central surface brightness against luminosity, Kormendy showed two relations, almost at right angles to each other: one for the $\mathrm{dE}$ galaxies and the other for the luminous elliptical galaxies. Similar diagrams using $\mu_{e}$, the surface brightness 
at the effective half-light radius $r_{e}$, or $\langle\mu\rangle_{e}$, the average surface brightness within $r_{e}$, also show two somewhat perpendicular relations (e.g., Capaccioli, Caon, \& D'Onofrio 1992). These differences are commonly interpreted as evidence for different formation mechanisms, resulting in the belief that a dichotomy exists between the $\mathrm{dE}$ and $\mathrm{E}$ galaxies. To understand, and in fact resolve, this apparent dichotomy, we must turn to the issue of galaxy structure.

In the past, some authors have restricted the radial extent of galaxy lightprofiles (excluding inner and outer parts; e.g, Burkert 1993) or adjusted the sky-background levels (e.g., Tonry et al. 1997) in order to make the $r^{1 / 4}$ model fit - such was the ingrained belief in this classic model. However, luminositydependent deviations from $r^{1 / 4}$ profiles had been known for some time (e.g., Capaccioli 1984, 1987; Michard 1985; Schombert 1986; Caldwell \& Bothun 1987; Kormendy \& Djorgovski 1989; Binggeli \& Cameron 1991; James 1991). Schombert (1986) recognized the inadequacy of the $r^{1 / 4}$ model for describing Es, since it only fits the middle $21<\mu_{B}<25$ part of bright galaxy profiles. Kormendy \& Djorgovski (1989) noted that the best $r^{1 / 4}$ fits were for Es with $M_{B} \sim-21 \mathrm{mag}$; brighter and fainter galaxies having a different logarithmic profile curvature than that of the $r^{1 / 4}$ model. It is these variations in the stellar distribution which have recently provided the key to understanding the true nature of the connection between the $\mathrm{dE}$ and $\mathrm{E}$ galaxies.

Sersic's (1968) $r^{1 / n}$ model can encompass both de Vaucouleurs' $r^{1 / 4}$ model and the exponential $(n=1)$ model — and a variety of other profile shapes - by varying its 'shape parameter' $n$. Analyzing a sample of 80 early-type galaxies in the Virgo and Fornax Clusters, Caon, Capaccioli, \& D'Onofrio (1993) and D'Onofrio, Capaccioli, \& Caon (1994) showed how the elliptical galaxy lightprofile shapes vary systematically with measurements of the half-light galactic radii and luminosity obtained independently of the fitted Sersic model. Additionally, numerous studies have demonstrated that $\mathrm{dE}$ galaxy profiles are not universally exponential, but rather are best fit with a range of Sersic profiles (i.e., $n$ is not always = 1; Davies et al. 1988; Cellone et al. 1994; Young \& Currie 1994; Durrell 1997; Jerjen \& Binggeli 1997; Binggeli \& Jerjen 1998; Graham \& Guzman 2003). The resulting trend between luminosity and lightprofile shape has begun to erase the dichotomy between dwarf and luminous ellipticals. To show how the remaining dichotomies can be eliminated, by explaining the apparently divergent behavior of $\mathrm{dE}$ and $\mathrm{E}$ galaxies in certain structural parameter diagrams, we will use the compilation of $249 \mathrm{dE}$ and $\mathrm{E}$ galaxies presented in Graham \& Guzman (2003) and shown here in Fig. 1.

Kormendy's (1985) plot of central surface brightness $\left(\mu_{0}\right)$ versus magnitude showed a large discontinuity and gap between the $\mathrm{dE}$ and $\mathrm{E}$ galaxies. However, there was an absence of galaxies with magnitudes around $M_{B}=-18 \pm 1$ in that sample, exactly where one might expect to see the two groups connect. If we remove galaxies with $M_{B}=-18 \pm 1$ from our plot of $\mu_{0}$ vs. $M_{B}$ (Fig. 1c), 


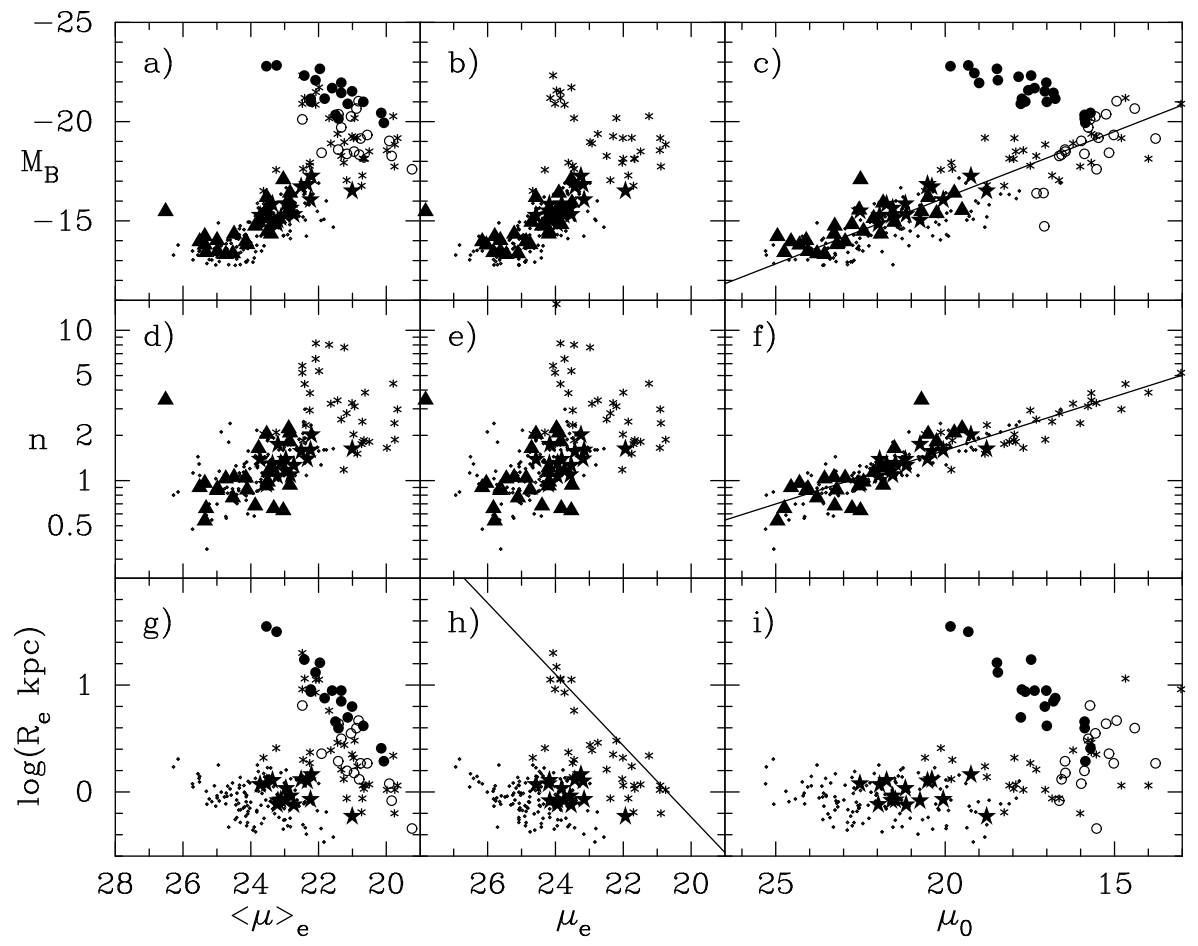

Figure 1. Mean surface brightness within $r_{e}\left(\langle\mu\rangle_{e}\right)$, surface brightness at $r_{e}\left(\mu_{e}\right)$, and central host galaxy surface brightness $\left(\mu_{0}\right)$ versus host galaxy magnitude $\left(M_{B}\right)$, global profile shape $(n)$, and half-light radius $\left(r_{e}\right)$. Due to biasing from the magnitude cutoff at $M_{B} \sim-13$, the line $M_{B}=(2 / 3) \mu_{0}-29.5$ in panel c) has been estimated by eye rather than by a linear regression routine. The line $\mu_{0}=22.8-14 \log (n)$ in panel $\mathrm{f}$ ) has also been estimated by eye. The line in panel h) has a slope of 3 and represents the Kormendy (1977) relation known to fit the luminous elliptical galaxies which define the panhandle of this complex distribution (Capaccioli \& Caon 1991; La Barbera et al. 2002). Dots represent dE galaxies from Binggeli \& Jerjen (1998), triangles are dE galaxies from Stiavelli et al. (2001), large stars are dE galaxies from Graham \& Guzman (2003), asterix are intermediate to bright E galaxies from Caon et al. (1993) and D'Onofrio et al. (1994), open circles represent the so-called "power-law" E galaxies from Faber et al. (1997), and the filled circles represent the "core" E galaxies from these same authors. Figure taken from Graham \& Guzman (2003). $H_{0}=70 \mathrm{~km} \mathrm{~s}^{-1} \mathrm{Mpc}^{-1}$.

we obtain a figure very much like Kormendy's (1985) Figure 3. Thus we can see that part of the "discontinuity" had arisen from an incomplete sampling of the intermediate-luminosity galaxies which fill in the apparent gap. Nevertheless, there is still an obvious change in slope in the overall $\mu_{0}-M_{B}$ relation. This can be explained with the observation that the most luminous elliptical galaxies possess partially evacuated "cores". Their observed central surface brightnesses are thought to be fainter than the original value due to the damage from coalescing supermassive black holes following a galaxy merger. As 


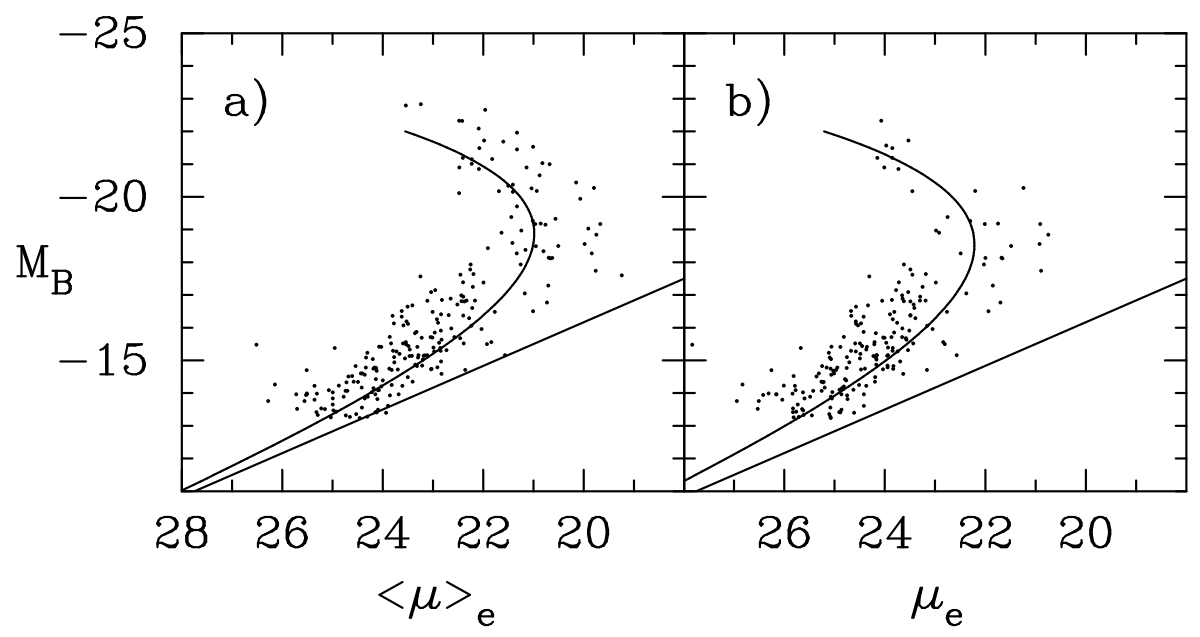

Figure 2. The curved lines show the predicted behavior of galaxies in the a) magnitude-mean surface brightness and b) magnitude-effective surface brightness diagrams. The straight line is from Fig. 1c; the data points are from Fig. 1a and $1 \mathrm{~b}$.

stressed in Graham \& Guzman (2003), the brighter galaxies lying perpendicular to the $\mu_{0}-M_{B}$ relation defined by the less luminous $\left(M_{B}>-20.5\right)$ elliptical galaxies are all "core" galaxies. The simplest explanation is that all elliptical galaxies follow a linear $\mu_{0}-M_{B}$ trend, except when core formation modifies the central surface brightness of the most luminous ellipticals. Since core formation is thought to be a later event, the initial mechanism(s) of $\mathrm{dE}$ and $\mathrm{E}$ galaxy formation are likely to be the same.

Another reason why E galaxies are thought to be different from $\mathrm{dE}$ galaxies is because they don't follow the same $M_{B}-\mu_{e}$ and $M_{B}-\langle\mu\rangle_{e}$ relations. Why are these relations apparently different for the $\mathrm{dE}$ and $\mathrm{E}$ galaxies? This, it turns out, has nothing to do with core formation but is due to the systematic changes in profile shape with galaxy magnitude. Even though many of the galaxies in Fig. 1a-c lack Sersic $n$ measurements, the relationship between magnitude and $n$ (e.g., Fig.10 in Graham \& Guzman 2003) can be used to determine a representative value of $n$ for a given $M_{B}$. From the Sersic model, we know that $\mu_{e}=\mu_{0}+1.086 b$ and $\langle\mu\rangle_{e}=\mu_{e}-2.5 \log \left[e^{b} n \Gamma(2 n) / b^{2 n}\right]$, where $b \sim 2 n-1 / 3$ (e.g., Graham \& Colless 1997). From this, the straight line in Fig. 1c transforms into the curved relationships between $M_{B}$ and $\mu_{e}$, and $M_{B}$ and $\langle\mu\rangle_{e}$ (Fig. 2). Thus, we can reproduce the observed correlations in Fig. 1a and 1b. The different slopes for the $\mathrm{dE}$ and $\mathrm{E}$ galaxy distributions in these diagrams are merely a consequence of a continuously varying profile shape with galaxy luminosity - they do not imply distinctly different galaxy 
formation processes for $\mathrm{dEs}$ and Es. Using $L=2 \pi r_{e}^{2}<I>_{e}$, it can be shown that the same mechanism is also behind the different slopes in the $\langle\mu\rangle_{e}-\log r_{e}$ and $M-\log r_{e}$ diagrams. A natural consequence is that the location of dEs and Es in Fundamental Plane type analyses that use $I_{e}$, or $\langle I\rangle_{e}$, will also be different.

\section{Acknowledgments}

We are grateful for funding provided by NASA through grants HST-AR08750.02-A and HST-AR-09927.01-A administered by the Space Telescope Science Institute. A.G. is also thankful for NSF funding administered by the American Astronomical Society's International Travel Grant Program.

\section{References}

Binggeli, B., \& Cameron, L.M. 1991, A\&A, 252, 27

Binggeli, B., \& Jerjen, H. 1998, A\&A, 333, 17

Binggeli, B., Sandage, A., \& Tarenghi, M. 1984, AJ, 89, 64

Burkert, A. 1993, A\&A, 278, 23

Caldwell, N., \& Bothun, G.D. 1987, AJ, 94, 1126

Caon, N., Capaccioli, M., \& D’Onofrio, M. 1993, MNRAS, 265, 1013

Capaccioli, M. 1984, in New Aspects of Galaxy Photometry, ed. J. Nieto, Springer-Verlag, p.53

Capaccioli, M. 1987, in Structure and Dynamics of Elliptical Galaxies, IAU Symp. 127, Reidel, Dordrecht, p.47

Capaccioli, M., \& Caon, N. 1991, MNRAS, 248, 523

Capaccioli, M., Caon, N., \& D’Onofrio, M. 1992, MNRAS, 259, 323

Cellone, S.A., Forte, J.C., \& Geisler, D. 1994, ApJS, 93, 397

Conselice, C.J. 2002, ApJ, 573, L5

Davies, J.I., et al. 1988, MNRAS, 232, 239

D’Onofrio, M., Capaccioli, M., \& Caon, N. 1994, MNRAS, 271, 523

Durrell, P. 1997, AJ, 113, 531

Faber, S.M., \& Lin, D.M.C. 1983, ApJ, 266, L17

Faber, S.M., et al. 1997, AJ, 114, 1771

Graham, A.W. 2002, ApJ, 568, L13

Graham, A.W., \& Colless, M. 1997, MNRAS, 287, 221

Graham, A.W., \& Guzman, R. 2003, AJ, 125, 2936

James, P. 1991, MNRAS, 250, 544

Jerjen, H., \& Binggeli, B. 1997, in The Nature of Elliptical Galaxies; The Second Stromlo Symposium, ASP Conf. Ser., 116, 239

Kormendy, J. 1977, ApJ, 218, 333

Kormendy, J. 1985, ApJ, 295, 73

Kormendy, J., \& Djorgovski, S. 1989, ARA\&A, 27, 235

La Barbera, F., et al. 2003, ApJ, 595, 127

Michard, R. 1985, A\&AS, 59, 205

Sandage, A., \& Binggeli, B. 1984, AJ, 89, 919

Schombert, J.M. 1986, ApJS, 60, 603

Sersic, J.L. 1968, Atlas de galaxias australes

Stiavelli, M., et al. 2001, AJ, 121, 1385 
Tonry J., Blakeslee J.P., Ajhar E.A., Dressler A. 1997, ApJ, 475, 399

Wirth, A., \& Gallagher, J.S. 1984, ApJ, 282, 85

Young, C.K., \& Currie, M.J. 1994, MNRAS, 268, L11 